\title{
Identification of gene signatures from RNA-seq data using Pareto-optimal cluster algorithm
}

\author{
Saurav Mallik ${ }^{1}$ and Zhongming Zhao ${ }^{1,2^{*}}$ \\ From The International Conference on Intelligent Biology and Medicine (ICIBM) 2018 \\ Los Angeles, CA, USA. 10-12 June 2018
}

\begin{abstract}
Background: Gene signatures are important to represent the molecular changes in the disease genomes or the cells in specific conditions, and have been often used to separate samples into different groups for better research or clinical treatment. While many methods and applications have been available in literature, there still lack powerful ones that can take account of the complex data and detect the most informative signatures.

Methods: In this article, we present a new framework for identifying gene signatures using Pareto-optimal cluster size identification for RNA-seq data. We first performed pre-filtering steps and normalization, then utilized the empirical Bayes test in Limma package to identify the differentially expressed genes (DEGs). Next, we used a multi-objective optimization technique, "Multi-objective optimization for collecting cluster alternatives" (MOCCA in R package) on these DEGs to find Pareto-optimal cluster size, and then applied k-means clustering to the RNA-seq data based on the optimal cluster size. The best cluster was obtained through computing the average Spearman's Correlation Score among all the genes in pair-wise manner belonging to the module. The best cluster is treated as the signature for the respective disease or cellular condition.

Results: We applied our framework to a cervical cancer RNA-seq dataset, which included 253 squamous cell carcinoma (SCC) samples and 22 adenocarcinoma (ADENO) samples. We identified a total of 582 DEGs by Limma analysis of SCC versus ADENO samples. Among them, 260 are up-regulated genes and 322 are down-regulated genes. Using MOCCA, we obtained seven Pareto-optimal clusters. The best cluster has a total of 35 DEGs consisting of all-upregulated genes. For validation, we ran PAMR (prediction analysis for microarrays) classifier on the selected best cluster, and assessed the classification performance. Our evaluation, measured by sensitivity, specificity, precision, and accuracy, showed high confidence.
\end{abstract}

Conclusions: Our framework identified a multi-objective based cluster that is treated as a signature that can classify the disease and control group of samples with higher classification performance (accuracy 0.935) for the corresponding disease. Our method is useful to find signature for any RNA-seq or microarray data.

Keywords: Gene signature, Cervical cancer, Pareto optimal clustering, K-means, Limma

\footnotetext{
*Correspondence: Zhongming.Zhao@uth.tmc.edu

${ }^{1}$ Center for Precision Health, School of Biomedical Informatics, The University

of Texas Health Science Center at Houston, Houston, TX 77030, USA

${ }^{2}$ Department of Biomedical Informatics, Vanderbilt University School of

Medicine, Nashville, TN 37232, USA
}

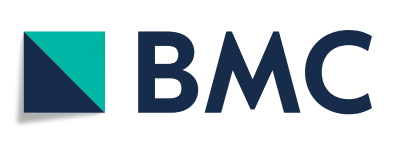

(c) The Author(s). 2018 Open Access This article is distributed under the terms of the Creative Commons Attribution 4.0 International License (http://creativecommons.org/licenses/by/4.0/), which permits unrestricted use, distribution, and reproduction in any medium, provided you give appropriate credit to the original author(s) and the source, provide a link to the Creative Commons license, and indicate if changes were made. The Creative Commons Public Domain Dedication waiver (http://creativecommons.org/publicdomain/zero/1.0/) applies to the data made available in this article, unless otherwise stated. 


\section{Background}

Detection of gene signatures from genomic data has been an important topic in medical domain during the last two decades. A "gene signature" can be stated as a single or a group of genes in a cell having a unique pattern of gene expression that is the consequence of either changed biological process or altered pathogenic medical terms.

Statistical analysis [1-7] is one of the most crucial techniques to determine differentially expressed transcripts [8-17] across a group of samples versus another group of samples. For RNA-seq data, proper selection of normalization and statistical test are very important, otherwise it might generate wrong $p$-value for each transcript. Voom normalization [18] is very useful for the RNA-seq data, whereas Limma tool $[2,19-21]$ is also useful for this kind of data.

Determining optimal cluster (module) number of the data is a challenging problem. In general, we set the number of clusters whenever we use any clustering algorithm. Thus, that is not optimal. Hence, as a result, this might increase the error-rate. Therefore, prior to use any clustering algorithm, it is necessary to estimate the Pareto-optimal cluster size using the combination of several clustering algorithms and various cluster validation indices as multi-objectives. It obviously reduces the errorrate whenever clustering on the underlying data. Of note, in case of the single-objective optimization problem [22], the superiority of a solution over other existing solutions can be produced very easily through the comparison of the scores of their objective functions. However, in the case of the multi-objective optimization problem [14, 15, 23-25] the goodness of a solution is generally identified through the dominance. The non-dominated solution set is basically a set of all the solutions which can not be dominated by none of the members of the solution set. Interestingly, the non-dominated set of the whole feasible decision space is stated as the Pareto-optimal set [26], whereas the boundary defined by the set of all the points that are mapped from the Pareto-optimal set, is denoted as the Pareto-optimal front [26]. MOCCA (Multi-objective optimization for collecting cluster alternatives) [27] is a latest robust estimator of Pareto-optimal cluster size through aggregating the best cluster numbers of various clustering algorithms and several cluster validation indices as the multi-objectives. MOCCA provides the ranking of the Pareto-optimal cluster sizes based upon their domination.

There are many bioinformatics approaches available for the gene signature identification. Mitra et al. identified gene-signature using a machine learning techniques including the Random forest and random survival forest algorithm for multiple myeloma [28]. Aziz et al. analyzed a microarray data using GeneSpring software and other existing $\mathrm{R}$ software and determined gene-signature in colorectal cancer [29]. Chen et al. applied a decisiontree analysis and survival analysis to identify gene signature on nonsmall-cell lung cancer [30]. Other related research works were [31-43]. So far, a very few attempts has been performed using through Pareto-optimal technique for gene signature identification. Basu et al. proposed Strength Pareto Evolutionary Algorithm for gene set selection [44]. Furthermore, neither pathway analysis nor gene-ontology analysis was carried out by them. Awad and Jong proposed another method of optimization of Spectral Signatures Selection through MOGA Multi-Objective Genetic Algorithm [45]. The overall performance of these methods are not so satisfactory. Hence, in this article, we developed a new framework of identifying gene signature using Pareto-optimal cluster identification for RNA-seq data. In this regard, we conducted some pre-filtering steps to remove the redundant feature from the dataset. Next, we utilized Voom normalization and then Limma tool to identify the differentially expressed genes. Thereafter, we applied MOCCA R tool on these differentially expressed genes using 12 objectives (i.e., kmeans.MCA, kmeans.Jaccard, kmeans.FM, kmeans.CQS, neuralgas.MCA, neuralgas.Jaccard, neuralgas.FM, neuralgas.CQS, single.MCA, single.Jaccard, single.FM and single.CQS) to estimate the Patero-optimal cluster size, and then applied k-means clustering through the optimal cluster size. The best cluster was obtained through computing the average Spearman's Correlation Score among all the genes in pair-wise manner belonging to the module. The best cluster is now treated as a signature for the respective disease. There are many ways to validate the gene signature. One of them is classification of the features (genes) belonging to the signature. For the purpose of validation, we applied PAMR (prediction analysis for microarrays) [46] classifier on all the features (genes) of selected best cluster, and computed the classification performances. In this work, we used TCGA cervical cancer dataset for experiment. We obtained high classification accuracy in the performance of the classifier. Finally, our method is useful to find signatures for any RNA-seq or similar kind of data.

\section{Methods}

In this article, we developed a framework for identifying the significant gene module from a RNA-seq expression dataset for a disease or specific cellular/physiological condition. The resultant gene modules will be integrated to obtain one final significant module. We used TCGA cervical cancer gene expression data and the phenotype data to test our method. The phenotype data was utilized to obtain the subtype of cervical cancer samples according to the Sample ID. 


\section{Finding differentially expressed genes}

First, we applied pre-filtering approaches (such as eliminating the genes having "all zeros", "NA value removal"). After that, we carried out gene-wise standardization. Thereafter, Voom normalization [18] and Limma R tool $[2,47]$ were then utilized consecutively to identify the differentially expressed genes. Limma used empirical Bayes test. As a result, we obtained a set of statistically significant genes. After that, we applied volcano plot using bi-filtering approaches ( $p$-value filtering and fold change filtering) consecutively. A up-regulated gene can be stated as a gene that had $p$-value less than 0.05 and fold change greater than 2, whereas a down-regulated gene be a gene having $p$-value less than 0.05 and fold change less than 0.5 .

\section{Pareto-optimal cluster selection}

Estimation of the Pareto-optimal cluster number of a data-profile is a challenging problem. Cluster validity

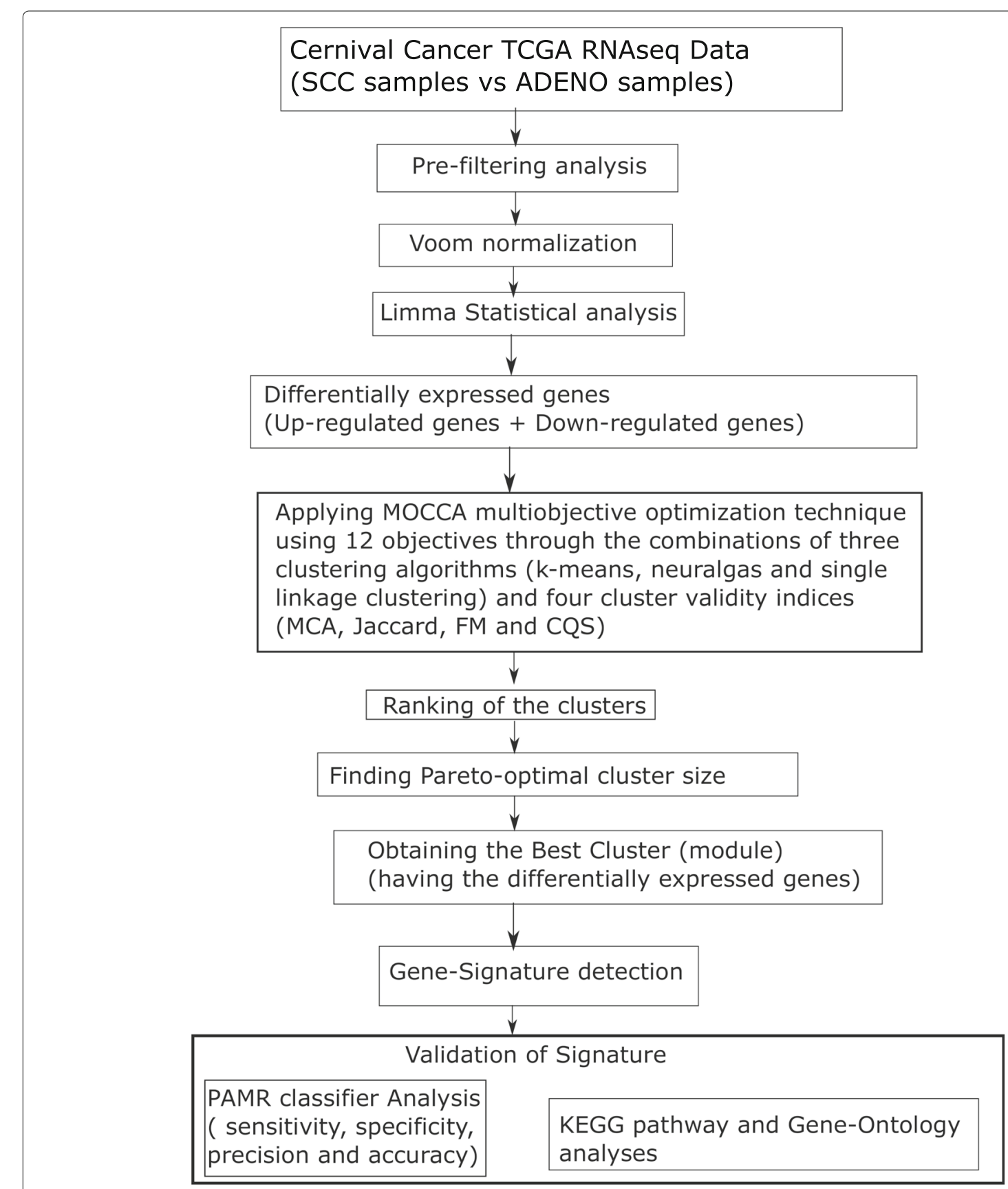

Fig. 1 Flowchart of the proposed framework 


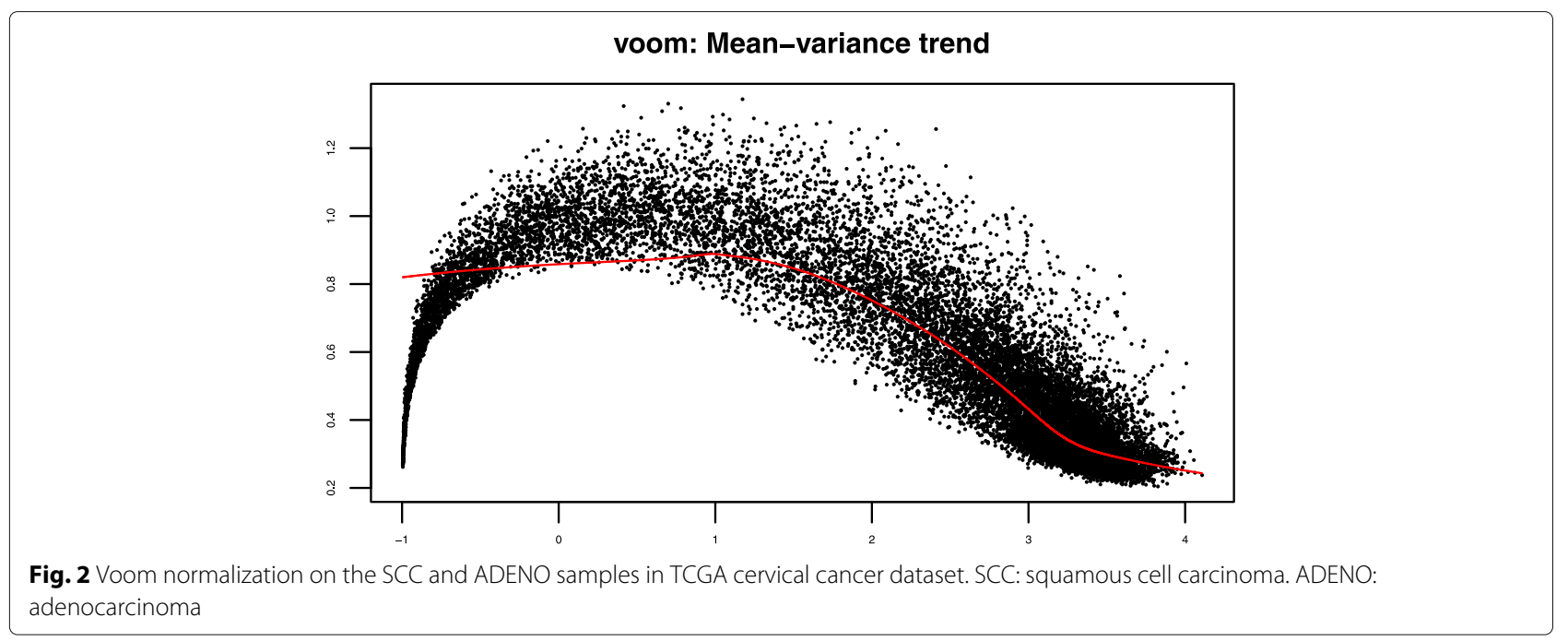

indices are basically developed to evaluate the performance of a clustering and can be applied to rank various cluster sizes. After finding the set of the differentially expressed genes (up-regulated genes and down-regulated genes), we applied a R package MOCCA [27] on the data of the differentially expressed genes to determine the optimal (robust) number of clusters. Bootstrapping approach is utilized to identify the robust cluster numbers depending upon several cluster validity indices. Of note, these estimations will differ based on the employed clustering technique as well as the cluster validation index. The central idea of MOCCA approach is to evaluate the robust (Pareto-optimal) cluster numbers through aggregation of the best cluster numbers of various clustering algorithms and various cluster validation indices in a multi-objective environment. In details, firstly, MOCAA

\section{Volcano plot}

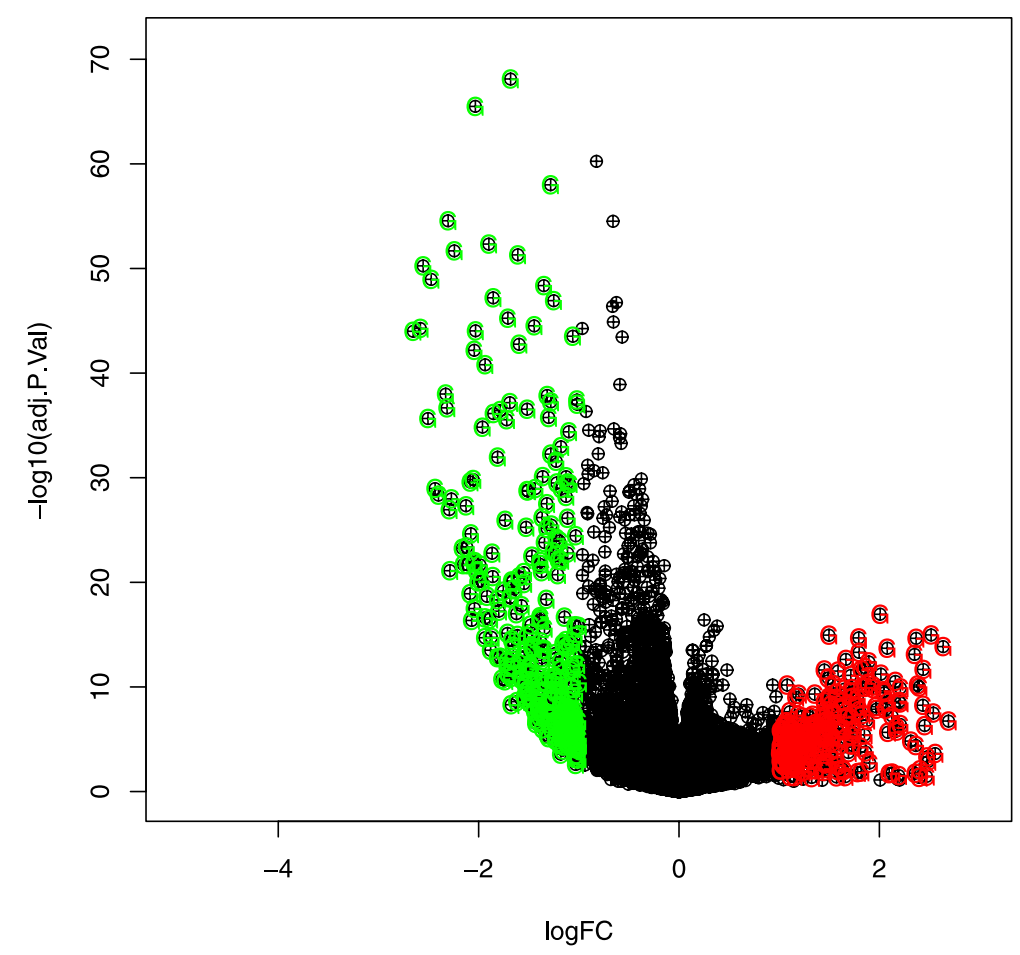

Fig. 3 Volcano plot for identifying up-regulated and down-regulated genes for ADENO vs. SCC subtypes in TCGA cervical cancer dataset 
Table 1 Twelve objectives in MOCCA and their values from the TCGA cervical cancer RNA-seq dataset

\begin{tabular}{ll}
\hline Objective & Objective value \\
\hline kmeans.MCA & 0.602 \\
kmeans.Jaccard & 0.509 \\
kmeans.FM & 0.608 \\
kmeans.CQS & 0.978 \\
neuralgas.MCA & 0.602 \\
neuralgas.Jaccard & 0.518 \\
neuralgas.FM & 0.613 \\
neuralgas.CQS & 0.979 \\
single.MCA & 0.551 \\
single.Jaccard & 0.349 \\
single.FM & 0.520 \\
single.CQS & 0.977 \\
\hline
\end{tabular}

conducts a multi-objective optimization to accumulate cluster alternatives. Next, it extracts $\mathrm{R}$ number of bootstrap samples from data-matrix. It computes the clustering for all specific cluster numbers $\mathrm{K}$ through the use of three clustering techniques (kmeans, single-linkage and neuralgas clustering). Thereafter, it utilizes various cluster validation indices (MCA, Jaccard, FM and CQS) to the clustering. Hence, a total of twelve objective functions (i.e., kmeans.MCA, kmeans.Jaccard, kmeans.FM, kmeans.CQS, neuralgas.MCA, neuralgas.Jaccard, neuralgas.FM, neuralgas.CQS, single.MCA, single.Jaccard, single.FM and single.CQS) were provided here to obtain Pareto optimal (robust) number of clusters. However, these outcomes (cluster validity indices) were then compared through determining the Pareto-optimal cluster sizes and ranking them depending upon their domination. Finally, a vector having the rank of Pareto-optimal cluster sizes had been provided based upon their domination along with the matrix whose each row was connected with a specified Pareto-optimal cluster size, and each cell entry referred to as how many objective functions it dominates the clustering of the other remaining cluster sizes. Specially, the Pareto-optimal cluster sizes were ranked according to the lowest number of objectives for which they dominated other remaining cluster sizes.

After determining the optimal number of clusters, we identified the cluster information of each participated gene through k-means clustering using the optimal cluster size.

\section{Ranking of the clusters}

After that, we computed Spearman's Correlation Coefficient score among the participating pairwise genes belonging to each individual resultant cluster and determined the average Spearman's Correlation Coefficient score of each cluster. The cluster whose average Spearman's Correlation Coefficient score was maximum, was chosen as best cluster. The combined gene set of the best cluster was here treated as a gene-signature.

\section{Signature selection and validation through classifier}

For the validation of the gene signature, we conducted a classification analysis containing all the features (genes) and all the samples having two groups (SCC and ADENO) using PAMR (prediction analysis for microarrays) tool [46].

Here, in details, we carried out 10-fold cross-validation and divided the data of all the genes belonging to the signature into training set and test set. Thereafter, we computed the threshold for which the error of the cross-validation would be minimum. PAMR classifier in "e107" R package [48] was then utilized using the training set and the resultant threshold for predicting the sample class of the test set. We repeat

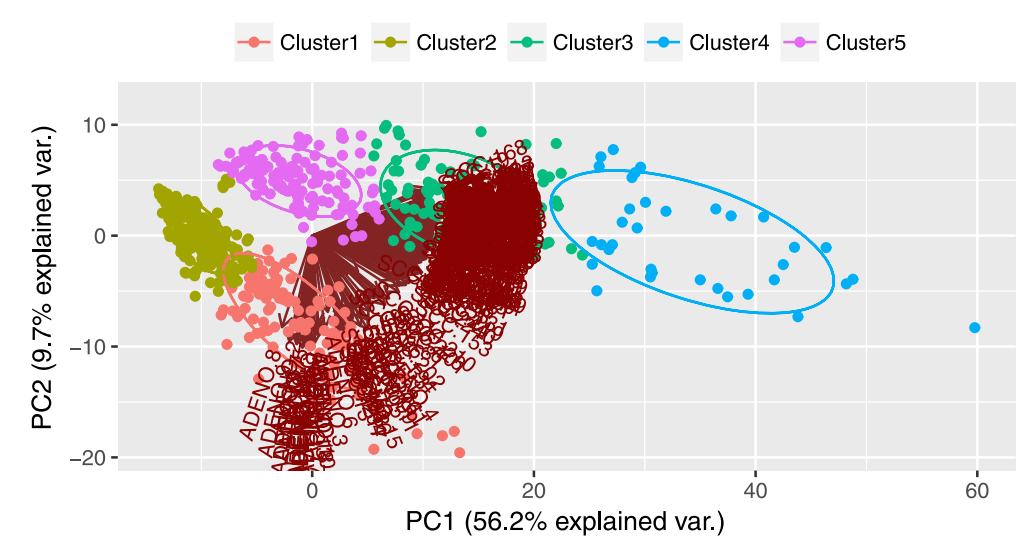

Fig. 4 Principal Component Analysis (PCA) of the clustering genes obtained from the comparison of ADENO with SCC subtypes in TCGA cervical cancer dataset 
the whole process for 10 times. We used four evaluation metrics to compute the overall performance of the classification. Finally, we computed average sensitivity, average specificity, average precision and average accuracy.

In addition to the classifier design, we performed KEGG pathway and Gene-Ontology (GO) analyses for the participating genes of the signature using DAVID database. Here, we picked up the KEGG pathways or Gene-Ontology terms whose enrichment $p$-values were less than 0.05 . Finally, we included a figure (Fig. 1) to represent all the steps of our method.

\section{Results}

In this section, we firstly describe the source of the cervical cancer dataset and then demonstrate the experimental results.

We used TCGA Cervical Squamous Cell Carcinoma and Endocervical Adenocarcinoma (TCGA-CESC) gene expression data (IlluminaHiSeq platform) using UCSC Xena browser [49]. We used Endocervical type of adenocarcinoma (ADENO) as control sample and cervical squamous cell carcinoma (SCC) as diseased/experimental samples. The number of control samples (ADENO) is 22 , whereas the number of experimental samples (SCC) is 253. The total number of genes is 20,530. We used these types of samples for comparison, the method can be applied to any pairs of samples (e.g. disease versus normal samples).

First of all, we collected the data from the aforementioned TCGA cervical dataset covering a total of the 20,530 genes. We then eliminated the redundant features using subsequent pre-filtering approaches as mentioned in "Pareto-optimal cluster selection" section. After prefiltering steps, we obtained a total of 19,709 genes. After that, we performed Voom normalization (in Fig. 2) and then Limma software consecutively to identify the differentially expressed genes. Thereafter, we utilized volcano plot (in Fig. 3) using bi-filtering approaches ( $p$-value filtering and fold change filtering) consecutively. A upregulated gene can be stated as a gene that had $p$-value less than 0.05 and fold change greater than 2 , whereas a down-regulated gene be a gene having $p$-value less than 0.05 and fold change less than 0.5 . As a result, we obtained a total number of 582 differentially expressed genes of which 260 are up-regulated genes and remaining 322 are down-regulated genes.

After finding the set of the up-regulated genes and down-regulated genes, we applied a R package MOCCA on the data of these genes to determine the Pareto-optimal (robust) number of cluster. As a result, we obtained 5 as Pareto-optimal cluster size. The objective values for the twelve objective functions are here represented in Table 1. After determining the optimal number of clusters $(n=5)$, we collected the cluster information of each participated gene through k-means clustering using the optimal cluster size. Of note, the number of up-regulated genes in these resultant five clusters are $0,4,95,35$ and 126, respectively, whereas the number of down-regulated genes in these resultant five clusters are 113, 209, 0, 0 and 0, respectively. Of note, the modules might be detected in such an iteration of k-means clustering using the fixed number of cluster numbers when the genes were likely to be in convergence during the run of the k-means clustering. Here it might be possible to obtain such clusters (modules) having imbalanced number of upregulated genes and downregulated genes.

Next, we calculated Spearman's Correlation Coefficient score among the participating pairwise genes belonging to each individual resultant cluster, and determined the average Spearman's Correlation Coefficient score of each cluster. The average Spearman's Correlation of the five clusters were $0.312,0.201,0.309,0.521$ and 0.211 , respectively. The fourth cluster having highest average Spearman's Correlation Coefficient score $(=0.521)$ was selected as "gene-signature". Of note, the gene-signature contained 35 differentially expressed genes of which all the genes were up-regulated. These up-regulated genes are AKR1B10, ANXA8, ANXA8L2, BNC1, CLCA2, CSTA, DSC3, FBXO27, FOXE1, GBP6, GJB6, GPR109A, GPR115, GPR87, IVL, KRT6A, KRT6B, KRT6C, LOC642587, NCCRP1, PKP1, PLAC2, RHCG, SBSN, SERPINB13, SERPINB2, SERPINB4, SOX15, SPRR1A, SPRR2A, SPRR2D, TMEM40, TMPRSS11D, TP63 and VSNL1. Of note, we performed Principal Component Analysis (PCA) plot on the resultant genes belonging to the resultant clusters. The clusters are nicely visible in Fig. 4.

Thereafter, as mentioned in "Signature selection and validation through classifier" section, we performed 10fold cross-validation and then ran PAMR classifier on all the features (genes) and all the samples having two groups (SCC and ADENO) belonging to the resultant signature. We repeat the whole process for 10 times. As a result, we obtained very good average accuracy $(=93.45 \%( \pm 0.297 \%))$ in the classification study. For details about all the evaluation criteria (average sensitivity, average specificity,

Table 2 Classification performance of the resultant gene signature having all the features and samples for the cervical cancer RNA-seq dataset

\begin{tabular}{ll}
\hline Evaluation criteria & Average(sd) \\
\hline Sensitivity & $0.934(0.27 \%)$ \\
Specificity & $0.941(2.20 \%)$ \\
Precision & $0.995(0.20 \%)$ \\
Accuracy & $0.935(0.30 \%)$ \\
\hline
\end{tabular}


Table 3 KEGG pathway and Gene Ontology (GO) enrichment analysis of the participating genes of the resultant gene signature for the cervical cancer RNA-seq dataset

\begin{tabular}{|c|c|c|}
\hline Gene set & $P$-value & Gene symbols \\
\hline GO:BPa: GO:0030216 keratinocyte differentiation & $1.01 \times 10^{-7}$ & SPRR1A, SPRR2D, SPRR2A, TP63, CSTA, IVL \\
\hline GO:CC $:$ GO:0001533 cornified envelop & $8.06 \times 10^{-7}$ & SPRR1A, SPRR2D, SPRR2A, CSTA, IVL \\
\hline GO:BP: GO:0018149 peptide cross-linking & $9.91 \times 10^{-7}$ & SPRR1A, SPRR2D, SPRR2A, CSTA, IVL \\
\hline GO:MF': GO:0005198 structural molecule activity & $1.30 \times 10^{-6}$ & $\begin{array}{l}\text { KRT6C, KRT6A, SPRR1A, SPRR2D, SPRR2A, CSTA, } \\
\text { IVL }\end{array}$ \\
\hline GO:BP: GO:0031424 keratinization & $5.44 \times 10^{-5}$ & SPRR1A, SPRR2D, SPRR2A, IVL \\
\hline GO:CC GO:0070062 extracellular exosome & $1.61 \times 10^{-4}$ & $\begin{array}{l}\text { KRT6C, KRT6A, GBP6, KRT6B, PKP1, NCCRP1, } \\
\text { RHCG, TMPRSS11D, AKR1B10, SBSN, SERPINBA, } \\
\text { SERPINB13, CSTA, IVL }\end{array}$ \\
\hline GO:BP: GO:0008544 epidermis development & $2.99 \times 10^{-4}$ & SPRR1A, SPRR2D, SPRR2A, BNC1 \\
\hline GO:BP: GO:0010951 negative regulation of endopeptidase activity & $8.41 \times 10^{-4}$ & SERPINB2, SERPINB4, SERPINB13, CSTA \\
\hline KEGG pathway: hsa05146:Amoebiasis & 0.002 & SERPINB2, SERPINB4, SERPINB13 \\
\hline GO:MF: GO:0030674 protein binding, bridging & 0.006 & SPRRTA, CSTA, IVL \\
\hline GO:MF GO:0004867 serine-type endopeptidase inhibitor activity & 0.009 & SERPINB2, SERPINB4, SERPINB13 \\
\hline GO:MF: GO:0002020 protease binding & 0.010 & SERPINB4, SERPINB13, CSTA \\
\hline GO:CC: GO:0045095 keratin filament & 0.011 & KRT6C, KRT6A, KRT6B \\
\hline GO:CC: GO:0005882 intermediate filament & 0.014 & KRT6C, KRT6A, PKP1 \\
\hline GO:BP: GO:0045104 intermediate filament cytoskeleton organization & 0.023 & KRT6C, KRT6A \\
\hline GO:BP: GO:0010466 negative regulation of peptidase activity & 0.026 & SERPINB4, CSTA \\
\hline GO:BP: GO:0030162 regulation of proteolysis & 0.032 & SERPINB4, SERPINB13 \\
\hline GO:CC: GO:0030057 desmosome & 0.038 & PKP1, DSC3 \\
\hline GO:BP: GO:0031069 hair follicle morphogenesis & 0.041 & FOXE1, TP63 \\
\hline GO:MF: GO:0004869 cysteine-type endopeptidase inhibitor activity & 0.049 & SERPINB13, CSTA \\
\hline
\end{tabular}

${ }^{a}$ Biological Processing, ${ }^{b}$ Cellular Components, ${ }^{c}$ Molecular Function

average precision, and average accuracy) are depicted in Table 2.

In addition, we performed gene set enrichment analysis using KEGG pathway and Gene-Ontology (GO) terms through DAVID online tool. As a result, we obtained one significant KEGG pathway (hsa05146:Amoebiasis having $p$-value 0.002) and nine significant GO:BPterms. These GO terms are GO:0030216 keratinocyte differentiation ( $p$-value $1.01 \times 10^{-7}$ ), GO:0018149 peptide cross-linking ( $p$-value $\left.9.91 \times 10^{-7}\right), \quad$ GO:0031424 keratinization ( $p$-value $\left.5.44 \times 10^{-5}\right)$, GO:0008544 epidermis development ( $p$-value $\left.2.99 \times 10^{-4}\right)$, GO:0010951 negative regulation of endopeptidase activity ( $p$-value $\left.8.41 \times 10^{-4}\right), \mathrm{GO} 0045104$ intermediate filament cytoskeleton organization ( $p$-value 0.023), GO:0010466 negative regulation of peptidase activity ( $p$-value 0.026 ), GO:0030162 regulation of proteolysis ( $p$-value 0.032 ) and GO:0031069 hair follicle morphogenesis ( $p$-value 0.041). We also identified five significant GO:CC-terms. These GO terms are GO:0001533 cornified envelop ( $p$-value $\left.8.06 \times 10^{-7}\right)$, GO:0070062 extracellular exosome
( $p$-value $\left.1.61 \times 10^{-4}\right), \quad$ GO:0045095 keratin filament ( $p$-value 0.011), GO:0005882 intermediate filament ( $p$-value 0.014) and GO:0030057 desmosome ( $p$-value 0.038). We found five significant GO:MFterms. These GO terms are GO:0005198 structural molecule activity ( $p$-value $\left.1.30 \times 10^{-6}\right)$, GO:0030674 protein binding, bridging ( $p$-value 0.006), GO:0004867 serine-type endopeptidase inhibitor activity ( $p$-value 0.009 ), GO:0002020 protease binding ( $p$-value 0.010 ) and GO:0004035 alkaline phosphatase activity ( $p$-value 0.049). For details, see Table 3.

\section{Discussion}

There are a lot of group lasso techniques (sglasso [50], flasso [50], etc.) available in the literature. But the objectives of these lasso techniques are different from our method. Lasso technique is basically a regression based study, whereas our method is Pareto optimal based clustering framework used only a single genomic or epigenetic data. So, we cannot compare our method with lasso based approaches. Furthermore, in the literature, there are a 
lot of co-expression based techniques for gene signature identification. But, the majority of the existing methods either follow a WGCNA module detection method or something like that where the generalized modules are not optimized [51, 52]. If the input threshold for the minimum number of module is changed, the number of modules is likely to change. To recover from the method, we first optimized the number of clusters in our method, and then used a standard clustering algorithm to find gene modules. Finally, we computed average Spearman's Correlation Coefficient of each module, and obtained the top ranked module as gene signature. Moreover, our method produces very high classification performance for the signature in terms of sensitivity, specificity, accuracy and precision. Hence, our method is beneficial in various aspects rather than other related existing methods.

\section{Conclusions}

Although there are many bioinformatics approaches available for the gene signature identification, the gene signature identification through Pareto-optimal technique has never been tried before. Therefore, in this article, we developed a new framework of identifying gene signature using Pareto-optimal cluster identification for RNA-seq data. In this regard, we conducted some pre-filtering steps to remove the redundant feature from the dataset. Next, we applied Voom normalization and then Limma statistical tool to find the differentially expressed genes consisting of up-regulated and down-regulated genes. Thereafter, we applied MOCCA R tool on these differentially expressed genes to estimate the Patero-optimal cluster size, and then applied k-means clustering through the optimal cluster size. The best cluster was obtained through computing the average Spearman's Correlation Score among all the genes in pair-wise manner belonging to the module/cluster. The best cluster is now treated as a signature for the respective disease. For validation, we applied PAMR classifier on all the genes of selected best cluster, and computed the classification performances. In this work, we used TCGA cervical cancer dataset for testing, and we found a 35 gene signature. We obtained high average classification accuracy $(=0.935( \pm 0.297 \%))$. The signature might be helpful for diagnosis of the disease. Finally, our method is useful to identify gene signature for any RNA-seq or similar kind of data.

\footnotetext{
Abbreviations

ADENO: Adenocarcinoma; BP: Biological processing; CC: Cellular component; CESC: Cervical squamous cell carcinoma and endocervical adenocarcinoma; DAVID: Database for annotation, visualization and integrated discovery; DEG: Differentially expressed genes; FM: F measure; GO: Gene-ontology; KEGG: Kyoto encyclopedia of genes and genomes; Limma: Linear models for microarray data; MCA: Multiple correspondence analysis; MF: Molecular function; MOCCA Multi-objective optimization for collecting cluster alternatives; PAM: Prediction analysis for microarrays; PCA: Principal component analysis; SCC: Squamous cell carcinoma; WGCNA: Weighted correlation network analysis
}

\section{Acknowledgments}

We thank the members in Bioinformatics and Systems Medicine Laboratory for the useful discussion.

\section{Funding}

Publication of this article was sponsored by National Institutes of Health grants (R01LM012806, R03DE028103, and R03DE027393) and the Cancer Prevention and Research Institute of Texas (CPRIT RP180734 and RP170668).

\section{Availability of data and materials}

The code is available at https://drive.google.com/open?id=1EAfFGGaD2lhmcjJUnS_rPoHMwial1qL

\section{About this supplement \\ This article has been published as part of BMC Systems Biology Volume 12 Supplement 8, 2018: Selected articles from the International Conference on Intelligent Biology and Medicine (ICIBM) 2018: systems biology. The full contents of the supplement are available online at https://bmcsystbiol.biomedcentral. com/articles/supplements/volume-12-supplement-8.}

\section{Authors' contributions}

SM has developed and implemented the proposed methodology, carried out experiments, written and revised the manuscript. ZZ conceived the project and participated in manuscript writing and revision. Both authors have read and approved the final manuscript.

\section{Authors' information}

S.M. is a post-doctoral fellow in the Bioinformatics and Systems Medicine Laboratory (BSML), Center for Precision Health, School of Biomedical Informatics, The University of Texas Health Science Center at Houston. He obtained his PhD in the Department of Computer Science \& Engineering from Jadavpur University, India. His research interest includes machine learning, statistical learning and bioinformatics.

Z.Z. is a professor in the Center for Precision Health, School of Biomedical Informatics, The University of Texas Health Science Center at Houston. He directs the Bioinformatics and Systems Medicine Laboratory (BSML) and UTHealth Cancer Genomics Core.

\section{Ethics approval and consent to participate}

Not applicable.

\section{Consent for publication}

Not applicable.

\section{Competing interests}

The authors declare that they have no competing interests.

\section{Publisher's Note}

Springer Nature remains neutral with regard to jurisdictional claims in published maps and institutional affiliations.

\section{Published: 21 December 2018}

\section{References}

1. Ritchie $M E$, et al. Limma powers differential expression analyses for $R$ NA-sequencing and microarray studies. Nucleic Acids Res. 2015;43(7):e47.

2. Smyth G. Linear Models and Empirical Bayes Methods for Assessing Differential Expression in Microarray Experiments. Stat Appl Genet Mol Biol. 2004;3(1):1-25.

3. He Z, Yu W. Stable feature selection for biomarker discovery. Comput Biol Chem. 2010;34(4):215-25.

4. Vickers A. Parametric Versus Non-Parametric Statistics in the Analysis of Randomized Trials with Non-Normally Distributed Data. BMC Med Res Methodol. 2005;5(35):1-12.

5. Bandyopadhyay S, et al. A Survey and Comparative Study of Statistical Tests for Identifying Differential Expression from Microarray Data. IEEE/ACM Trans Comput Biol Bioinform. 2013;11(1):95-115.

6. Hogg RV, Ledolter J. Engineering Statistics. New York: MacMillan Pub Co; 1987.

7. Vapnik VN. The Nature of Statistical Learning Theory. second edition. New York: Springer; 2000. 
8. Mallik S, Zhao Z. TrapRM: Transcriptomic and Proteomic Rule Mining using Weighted Shortest Distance Based Multiple Minimum Supports for Multi-Omics Dataset. IEEE Int Conf Bioinforma Biomed (BIBM). 2017;2187-94. Kansas City, https://doi.org/10.1109/BIBM.2017.8217997.

9. Mallik S, Zhao Z. ConGEMs: Condensed Gene Co-Expression Module Discovery Through Rule-Based Learning and Its Application to Lung Squamous Cell Carcinoma. Genes. 2017;9(1):7.

10. Mallik S, Zhao Z. Towards integrated oncogenic marker recognition through mutual information-based statistically significant feature extraction: an ARM-based study on leukemia expression and methylation profiles. Quant Biol. 2017;5(4):302-27.

11. Mallik S, et al. IDPT: Insights into Potential Intrinsically Disordered Proteins Through Transcriptomic Analysis of Genes for Prostate Carcinoma Epigenetic Data. Gene. 2016;586(2016):87-96.

12. Bandyopadhyay S, Mallik S. Integrating Multiple Data Sources for Combinatorial Marker Discovery: A Study in Tumorigenesis. IEEE/ACM Trans Comput Biol Bioinform. 2018;15:2.

13. Bhattacharjee $S$, et al. Combinatorial Control of Gene Expression. BMC Syst Biol. 2013;407263:1-11.

14. Mukhopadhyay A, Mandal M. Identifying Non-redundant Gene Markers from Microarray Data: A Multiobjective Variable Length PSO-based Approach. IEEE/ACM Trans Comput Biol Bioinform. 2014;11(6):1-14.

15. Mallik S, et al. Multi-Objective Optimization Approaches in Biological Learning System on Microarray Data. In: Mondal JK, et al, editors. Multi-Objective Optimization. Singapore: Springer; 2018. p. 159-180.

16. Alon $U$, et al. Broad patterns of gene expression revealed by clustering analysis of tumor and normal colon tissues probed by oligonucleotide arrays. Proc. of the National Academy of Sciences. 1999. 96(12):6745-50. USA: Cell Biology.

17. Li J, et al. A new framework for identifying differentially expressed genes. Pattern Recogn. 2007;40(11):3249-62.

18. Law CW, et al. Voom: Precision weights unlock linear model analysis tools for RNA-seq read counts. Genome Biol. 2014;15(2):R29.

19. Maulik U, et al. Analyzing Gene Expression and Methylation Data Profiles using StatBicRM: Statistical Biclustering-based Rule Mining. Plos One. 2015;10(4):e0119448.

20. Mallik S, et al. RANWAR: Rank-Based Weighted Association Rule Mining from Gene Expression and Methylation Data. IEEE T Nanobiosci. 2015;14(1):59-66

21. Mallik S, et al. Integrated Analysis of Gene Expression and Genome-wide DNA Methylation for Tumor Prediction: An Association Rule Mining-based Approach. Proc. IEEE Symposium on Computational Intelligence in Bioinformatics and Computational Biology (CIBCB), IEEE Symposium Series on Computational Intelligence - SSCI. Singapore; 2013. pp. 120-7. https://doi.org/10.1109/CIBCB.2013.6595397.

22. Anderberg MR. Cluster Analysis for Applications. SIAM Rev. 1975;17(3): 580-2.

23. Maulik U, et al. Combining Pareto-optimal clusters using supervised learning for identifying co-expressed genes. BMC Bioinformatics. 2009;10(27).

24. Bandyopadhyay S, et al. Multiobjective Genetic Clustering for Pixel Classification in Remote Sensing Imagery. IEEE Geosci Remote Sens. 2007;45(5):1506-11

25. Strehl A, Ghosh J. Cluster ensembles - a knowledge reuse framework for combining multiple partitions. J Mach Learn Res. 2002;3:583-617.

26. Runkler TA. Pareto Optimality of Cluster Objective and Validity Functions. London: IEEE Int on Fuzzy Systems Conf (FUZZ-IEEE 2007); 2007. pp. 23-26.

27. https://cran.r-project.org/web/packages/MOCCA/MOCCA.pdf. Accessed 12 Dec 2017.

28. Mitra AK, et al. A gene expression signature distinguishes innate response and resistance to proteasome inhibitors in multiple myeloma. Blood Cancer J. 2017;7:e581.

29. Aziz NAA, et al. A 19-Gene expression signature as a predictor of survival in colorectal cancer. BMC Med Genet. 2016;20169:58.

30. Chen HY, et al. A Five-Gene Signature and Clinical Outcome in NonSmall-Cell Lung Cancer. N Engl J Med. 2017;356:11-20.

31. Sen S, et al. Detecting TF-MiRNA-Gene Network Based Modules for $5 \mathrm{hmC}$ and $5 \mathrm{mC}$ Brain Samples: A Intra- and Inter-Species Case-Study Between Human and Rhesus. BMC Genet. 2018;19:9.

32. Zheng $\mathrm{S}$, et al. Integrative network analysis identifies key genes and pathways in the progression of hepatitis $C$ virus induced hepatocellular carcinoma. BMC Med Genomics. 2011;4:62.
33. Mallik S, Maulik U. Module-Based Knowledge Discovery for Multiple-Cytosine-Variant Methylation Profile. Singapore: Soft Computing for Biological Systems, Springer; 2018.

34. Aqil M, et al. The HIV Nef protein modulates cellular and exosomal miRNA profiles in human monocytic cells. J Extracell Vesicles. 2014;3:23129.

35. Aqil M, et al. Transcriptomic Analysis of mRNAs in Human Monocytic Cells Expressing the HIV-1 Nef Protein and Their Exosomes. BioMed Res Int. 2015;492395:1-10.

36. Ruiza R, et al. Incremental wrapper-based gene selection from microarray data for cancer classification. Pattern Recognit. 2006;39(12):2383-92.

37. Xiong $\mathrm{M}$, et al. Biomarker identification by feature wrappers. Bioinformatics. 2001;11(11):1878-87.

38. Glaab E, et al. Using Rule-Based Machine Learning for Candidate Disease Gene Prioritization and Sample Classification of Cancer Gene Expression Data. PLoS One. 2012;7(7):e39932.

39. Cheng F, et al. A Gene Gravity Model for the Evolution of Cancer Genomes: A Study of 3,000 Cancer Genomes across 9 Cancer Types. PLOS Comput Biol. 2015;1:25.

40. Mallik S, et al. Identifying Epigenetic Biomarkers using Maximal Relevance and Minimal Redundancy Based Feature Selection for Multi-Omics Data. IEEE T Nanobiosci. 2017;16(1):3-10.

41. Shi $Z Q$, et al. Identification of effective combinatorial markers for quality standardization of herbal medicines. J Chromatogr A. 2014;1345:78-85.

42. Rakha EA, et al. Combinatorial biomarker expression in breast cancer. Breast Cancer Res Treat. 2010;120(2):293-308.

43. Huang HC, et al. Discovering Disease-specific Biomarker Genes for Cancer Diagnosis and Prognosis. Technol Cancer Res Treat. 2010;9(3):219-30.

44. Basu S, et al. Strength Pareto Evolutionary Algorithm based Gene Subset Selection. Chirala, India: Int. Conf. On Big Data Analytics and Computational Intelligence (ICBDACI); 2017.

45. Awad MM, Jong KD. Optimization of Spectral Signatures Selection Using Multi-Objective Genetic Algorithms. New Orleans: IEEE Congress on Evolutionary Computation (CEC); 2011.

46. Tibshirani R, et al. Diagnosis of multiple cancer types by shrunken centroids of gene expression. Proc Natl Acad Sci. 2002;99(10):6567-72.

47. Mallik S, et al. Integrated Statistical and Rule-Mining Techniques for DNA Methylation and Gene Expression Data Analysis. JAISCR. 2013;3(2):101-15.

48. Meyer D, et al. Misc functions of the Department of Statistics (e1071), TU Wien. R Package Version. 1.6-8. 2017 https://cran.r-project.org/web/ packages/e1071/e1071.pdf. Accessed 9 Jan 2018.

49. https://xenabrowser.net/datapages/?cohort=TCGA\%20Cervical \%20Cancer\%20(CESC). Accessed 9 Jan 2018.

50. https://cran.r-project.org/web/packages/sglasso/sglasso.pdf. Accessed 9 Jan 2018.

51. Van Dam S, et al. Gene co-expression analysis for functional classification and genedisease predictions. Brief Bioinform. 2017;bbw139:2017.

52. Lu X, et al. Driver pattern identification over the gene co-expression of drug response in ovarian cancer by integrating high throughput genomics data. Sci Rep. 2017;7:16188.

\section{Ready to submit your research? Choose BMC and benefit from}

- fast, convenient online submission

- thorough peer review by experienced researchers in your field

- rapid publication on acceptance

- support for research data, including large and complex data types

- gold Open Access which fosters wider collaboration and increased citations

- maximum visibility for your research: over $100 \mathrm{M}$ website views per year

At BMC, research is always in progress.

Learn more biomedcentral.com/submissions 